\title{
2-Y-E1-3 YIA
}

\section{1,2-Naphthoquinone functions as an activator of the EGF receptor via covalent modification of a specific lysine residue}

\author{
Kengo Nakahara ${ }^{1}$, Tomoki Tsuchida ${ }^{1}$, Yumi Abiko², Yoshito Kumagai ${ }^{2}$, Takashi Uehara ${ }^{1}$ \\ ${ }^{I}$ Department of Medical pharmacology, Graduate School of Medicine, Dentistry, and Pharmaceutical Sciences, \\ Okayama University, ${ }^{2}$ Environmental Biology Laboratory, Faculty of Medicine, University of Tsukuba
}

[Background] The epidermal growth factor receptor (EGFR) is the most intensively investigated receptor tyrosine kinase whose mutation or modification leads to abnormal self-activation, which plays a critical role in carcinogenesis. Although environmental air pollutants, which are associated with cancer and respiratory diseases, can activate EGFR, the detailed mechanisms remain unknown. In this study, we addressed to clarify the mechanisms by which the air pollutant 1,2-naphthoquinone (1,2-NQ) disrupts EGFR-Akt signaling and promotes oncogenic effects.

[Methods] Phosphorylated forms of Akt, EGFR, IGF-IR, and Insulin receptor were detected by western blotting using specific antibodies. The modification sites in EGFR were identified by LC-MS/MS analysis. Cell viability was assessed by WST-8 assay.

[Results] We found that 1,2-NQ stimulates the phosphorylation of EGFR, but not IGF-IR and Insulin receptor in A549 cells. LC-MS/MS analysis revealed that 1,2-NQ forms chemical modification with EGFR at ligand-binding domain I, Lys80. In addition, phosphorylation of Akt was observed by treatment with 1,2-NQ in a concentrationdependent manner. Activation of Akt by $1,2-\mathrm{NQ}$ was suppressed by pretreatment with tyrphostinA25, a specific EGFR tyrosine kinase inhibitor. Moreover, 1,2-NQ exerted the cytoprotective effect against for serum deprivationinduced cell death through the activation of EGFR-Akt signaling.

[Conclusions] These findings showed that 1,2-NQ directly activates EGFR-Akt signaling via chemical modification of EGFR. 